Stoa

Vol. 11, no. 21, 2020, pp. 98-109

ISSN 2007-1868

\title{
¿ES LA LÓGICA DE TÉRMINOS UNA LÓGICA LIBRE?
}

Is Terminist Logic a Free Logic?

José Martín CAstro Manzano

Universidad Popular Autónoma de Puebla

josemartin.castro@upaep.mx

RESUMEN: Los estudios sobre lógica libre están más o menos consolidados, dada la cantidad de estudios sobre lógicas libres. Esta situación contrasta con los estudios sobre lógicas de términos, ya que estos son de reciente creación. Dada esta asimetría, en este trabajo exploramos un nexo entre ambas lógicas con el propósito de sugerir que la lógica de términos de Sommers y Englebretsen se comporta como una lógica libre.

PALABRAS CLAVE: Lógica libre - Lógica de términos · Lógica clásica · Lógica noclásica.

ABSTRACT: Studies on free logic are more or less consolidated, given the number of studies on free logics. This situation contrasts with the status of studies on term logics, since these are of recent creation. Given this asymmetry, in this paper we explore a link between these two logics in order to suggest that the term logic developed by Sommers and Englebretsen behaves like a free logic.

KEY WORDS: Free logic · Term logic $\cdot$ Classical logic $\cdot$ Non-classical logic.

\section{Introducción}

Usualmente decimos que una lógica es una lógica libre cuando está libre de supuestos ontológicos; la lógica libre, en particular, es una lógica que es libre con respecto a ciertos ítems singulares, como los nombres propios, por ejemplo. Así se entiende la propuesta de Karel Lambert (1958, 1960, 1963): la lógica libre es una desviación ( $c f r$. Palau 2002, p. 36) de la lógica clásica de primer orden que resulta de la adición de ítems singulares inexistentes, como veremos más adelante. 
También en la década de 1960 otro filósofo, Fred Sommers, reconsideró la idea de que la lógica tiene que lidiar con inferencias en lenguaje natural, lo que le llevó a revisar las virtudes de la sintaxis de términos aristotélica. Esta revisión produjo resultados originales en tres áreas: ontología, filosofía del lenguaje y lógica ( $c f r$. Sommers 2005). En ontología dio lugar a una teoría de categorías; en filosofía del lenguaje, a una teoría sobre la estructura categórica del lenguaje; y en lógica, a una teoría lógica que hace uso de términos en lugar de elementos de la lógica clásica de primer orden, como también veremos renglones abajo. Pues bien, no es una sorpresa que los estudios sobre lógica libre estén más o menos consolidados, dada la cantidad de estudios representativos sobre lógicas libres (Jaśkowski 1934, Mostowski 1951, Hailperin 1953, Lejewski 1954, Leonard 1956, Lambert 1958, Lambert 1960, Lambert 1963); lo cual-tampoco sorprende-contrasta con el estatuto de los estudios sobre las lógicas de términos á la Sommers, ya que estos son de reciente creación.

Dada esta asimetría, en este trabajo exploramos un nexo entre ambas lógicas con el propósito de sugerir que la lógica de términos de Sommers (y Englebretsen) se comporta como una lógica libre. Si nuestra intuición es correcta, esto aportaría un argumento adicional para considerar a la lógica de Sommers y Englebretsen como una lógica tradicional que no es clásica ( $c f r$. Englebretsen 1996, Oderberg 2005, Woods e Irvine, 2004).

Para alcanzar nuestra meta procedemos de la siguiente manera: primero hacemos una presentación elemental de la lógica libre, posteriormente exponemos una introducción básica a la lógica de términos de Sommers y Englebretsen y, por último, exploramos en qué sentido esta última puede considerarse como una lógica libre.

\section{La lógica libre}

Como la lógica libre es una modificación de la lógica clásica de primer orden (FOL, por First Order Logic) es conveniente empezar con una breve caracterización de esta mediante la exposición su lenguaje y su base deductiva.

Pues bien, el vocabulario de FOL se define a través de cuatro conjuntos: el de variables individuales, $V A R=\left\{x_{0}, x_{1}, x_{2} \ldots\right\}$; el de constantes individuales, $C O N S=\left\{c_{0}, c_{1}, c_{2}, \ldots\right\}$; el de relaciones $n$-arias, $R E L=\left\{R_{0}^{n}, R_{1}^{n}, R_{2}^{n}, \ldots\right\}$; y el de constantes lógicas, $C O N=\neg, \wedge, \vee, \subset, \equiv, \forall, \exists$. Dado este vocabulario, una constante o una variable individual determinan un término t. Si $t_{1} \ldots t_{n}$ son términos y $R_{0}^{n}$ es una relación $n$-aria, entonces $R_{0}^{n} t_{1} \ldots t_{n}$ es una fórmula atómica. Si $A$ y $B$ son fórmulas atómicas entonces $\neg A, A \wedge B, A \vee B, A \subset B, \mathrm{y}$ 
$A \equiv B$ son fórmulas. Y si $A$ es una fórmula y $x$ es una variable entonces $\forall x A$, $\exists x A$ son fórmulas también.

Una interpretación en esta lógica es un par $\langle D, v>$ tal que $D \neq \varnothing$ es un dominio de cuantificación y $v$ es una función que asigna elementos del lenguaje al dominio. Así, si $c$ es una constante, $v(c) \in D$; si $R^{n}$ es una relación $n$-aria, $v\left(R^{n}\right) \subseteq D^{n} ; \mathrm{y}$

$$
\begin{gathered}
v(\forall x A)=1 \text { si y sólo si para todo } d \in D, v\left(A\left[x / c_{d}\right]\right)=1, \text { y } 0 \text { de otro modo; y } \\
v(\exists x A)=1 \text { si y sólo si para algun } d \in D, v\left(A\left[x / c_{d}\right]\right)=1 \text { y } 0 \text { de otro modo. }
\end{gathered}
$$
(nota)

Ahora bien, una lógica libre es una lógica de primer orden que no asume ciertos principios de FOL, por tanto, el vocabulario y la sintaxis de la lógica libre (FFOL, por Free First Order Logic) son los mismos que los de FOL; sin embargo, FFOL reserva un predicado especial de existencia que se especifica de la siguiente manera:

$$
E ! t={ }_{d} f \exists x \cdot x=t,
$$

y que lee como " $t$ existe."

Una interpretación en FFOL se define por una terna $\left\langle D, D^{*}, v^{*}\right\rangle$ tal que $D \neq \varnothing, D^{*} \subseteq D$ es posiblemente vacío y $v^{*}$ es una función que se comporta como la $v$ de FOL pero que hace $v^{*}(E)=D^{*}$. Esto significa que $D$ es el dominio de cuantificación de todos los objetos pero $D^{*}$ es un subconjunto especial de todos los objetos que existen, por lo que $E$ ! $t$ es verdadero si $t$ denota un miembro de $D^{*}$ pero es falso si no lo hace. Así, se puede decir, por ejemplo, que $D$ contiene ítems singulares como Guillermo de Baskerville (digamos $g_{1}$ ) y Guillermo de Occam (digamos $g_{2}$ ), pero mientras $g_{2} \in D$ y $g_{2} \in D^{*}, g_{1} \in D$ pero $g_{1} \notin D^{*}$. Por tanto, como es de esperarse, las condiciones de verdad para una lógica como esta son similares a las de FOL pero con ciertas variaciones, a saber:

$$
\begin{gathered}
v(\forall x A)=1 \text { si y sólo si para todo } d \in D^{*}, v\left(A\left[x / c_{d}\right]\right)=1, \text { y 0de otro modo; } \\
v(\exists x A)=1 \text { si y sólo si para algún } d \in D^{*}, v\left(A\left[x / c_{d}\right]\right)=1, \text { y 0de otro modo. }
\end{gathered}
$$

Como consecuencia, la lógica libre permite incorporar el uso de ítems singulares inexistentes mediante el rechazo de aquellas reglas de inferencia cuya 
validez depende de la suposición de que tales ítems denotan a miembros del dominio de cuantificación, a saber, la regla de eliminación del cuantificador universal $(E \forall)$ y la regla de introducción del cuantificador existencial $(I \exists)$.

Para ilustrar este punto observemos la regla $E \forall$ en FOL:

$$
\begin{aligned}
& \forall x A x \\
& \vdash A[t / x]
\end{aligned}
$$

Esta regla es válida en FOL, dado que el dominio de cuantificación no es vacío, pero no puede ser válida en FFOL puesto que aun si todo ítem singular en el dominio de cuantificación satisface $A$, si ocurre que $t$ no denota a un miembro del dominio, entonces la conclusión $A[t / x]$ puede ser falsa aunque $\forall x A x$ sea verdad. Por ejemplo, sea $A x:=x$ es hijo de una persona y $t:=$ Bartleby (el escribiente).

Algo similar ocurre con la regla $I \exists$ :

$$
\begin{aligned}
& \text { At } \\
& \vdash \exists x A[t / x]
\end{aligned}
$$

Esta regla es válida en FOL pero no lo es en FFOL porque si $t$ no denota un objeto del dominio de cuantificación, entonces la verdad de $A$ no garantiza que exista un ítem en el dominio que satisfaga la expresión $A[t / x]$. Por ejemplo, sea $t:=$ Klaatu y $A x:=x$ emitió la frase 'Klaatu barada nikto.'

Consecuentemente, para distinguir a los ítems que denotan de los que no, FFOL ofrece versiones debilitadas de $E \forall$ y $I \exists$ como sigue:

\begin{tabular}{|l|l|}
\hline$E \forall$ & $I \exists$ \\
\hline$\forall \forall x A x$ & $A t$ \\
\hline$E ! t$ & $E ! t$ \\
\hline$\vdash A[t / x]$ & $\vdash \exists x A[t / x]$ \\
\hline
\end{tabular}

Tabla 1: Versiones de $E \forall$ y $I \exists$ en FFOL

Ciertamente, aunque esta caracterización de la lógica libre es sumatoria y requiere mayor precisión ( $c f r$. Bencivenga 2002), es suficiente para nuestros fines. 


\section{La lógica de términos de Sommers y Englebretsen}

La lógica de términos funtoriales de Sommers $(1975,1984,1989)$ y Englebretsen (1996, 2017) (TFL, por Term Functor Logic) es una lógica que representa la silogística (vide Apéndice A) usando términos en lugar de elementos lingüísticos de primer orden como variables individuales o cuantificadores ( $c f r$ Quine 1971, Noah 1980, Kuhn 1983), por lo que TFL no es una lógica de primer orden.

De acuerdo con esta propuesta terminista las cuatro proposiciones categóricas de la silogística pueden representarse mediante la siguiente sintaxis, donde “-” indica que un término está distribuido y “+” indica que un término no está distribuido (Englebretsen 1996, p.159):

\begin{tabular}{|l|l|l|}
\hline Proposición & Silogística & TFL \\
\hline \hline Todo S es P. & SaP & $-\mathrm{S}+\mathrm{P}$ \\
\hline Todo S no es P. & SeP & - S-P \\
\hline Algún S es P. & SiP & + S+P \\
\hline Algún S no es P. & SoP & + S-P \\
\hline
\end{tabular}

Tabla 2: Sintaxis de TFL para la silogística

Dada esta representación, TFL ofrece una regla de inferencia para la silogística: una conclusión se sigue válidamente de un conjunto (finito) de premisas si y sólo si i) la suma de las premisas es algebraicamente igual a la conclusión y ii) el número de conclusiones con cantidad particular (viz., cero o uno) es igual al número de premisas con cantidad particular (Englebretsen 1996, p. 167). Así, por ejemplo, si consideramos un silogismo válido, digamos un Barbara, podemos ver cómo la aplicación de este método produce la conclusión correcta:

\begin{tabular}{|l|l|}
\hline Proposición & TFL \\
\hline \hline 1. Todos los mamíferos son animales. & $-\mathrm{M}+\mathrm{A}$ \\
\hline 2. Todos los perros son mamíferos. & $-\mathrm{P}+\mathrm{M}$ \\
\hline$\vdash$ Todos los perros son animales. & $-\mathrm{P}+\mathrm{A}$ \\
\hline
\end{tabular}

Tabla 3: Un silogismo Barbara 
En el ejemplo anterior podemos ver claramente cómo es que funciona este método: i) si sumamos las premisas obtenemos la expresión $(-M+A)+(-P+$ $M)=-M+A-P+M=-P+A$, de tal modo que la suma de las premisas es algebraicamente igual a la conclusión, y la conclusión es igual a $-P+A$, en lugar de $+A-P$, porque por la condición ii) el número de conclusiones con cantidad particular (cero en este ejemplo) es igual al número de premisas con cantidad particular (cero en este ejemplo).

Como se puede apreciar, esta regla es una versión algebraica del dictum de omni et nullo. El mismo Sommers llama a esta regla "DON" y la estipula de la siguiente manera (Sommers 1975, 1989): sea $\mathrm{P}(\mathrm{M})$ una proposición con una ocurrencia positiva de $\mathrm{M}$ (i.e. $\mathrm{M}$ no aparece distribuido) en el ambiente proposicional $\mathrm{P}$, y sea $\mathrm{P}$ '(-M) una proposición en la cual $\mathrm{M}$ ocurre negativamente (i.e. $\mathrm{M}$ aparece distribuido) en el ambiente P', entonces:

\begin{tabular}{|l|}
\hline 1. $\mathrm{P}^{\prime}(-\mathrm{M})$ Premisa donante \\
\hline 2. $\mathrm{P}(\mathrm{M})$ Premisa anfitriona \\
\hline$\vdash \mathrm{P}\left(\mathrm{P}^{\prime}\right)$ Conclusión \\
\hline
\end{tabular}

Tabla 4: DON

Como se puede apreciar, la conclusión surge de sumar las premisas de tal manera que se cancela el término medio positivo en $\mathrm{P}(\mathrm{M})$, reemplazando tal término por P'; en otras palabras, todo lo que se afirma o niega de un todo (premisa donante) puede ser afirmado o negado de una parte (premisa anfitriona).

Contrariamente a lo que se podría pensar, TFL no está limitada a la inferencia silogística, ya que esta aproximación algebraica es capaz de representar y modelar inferencias con proposiciones relacionales (Tabla 5), singulares (Tabla 6) y compuestas (Tabla 7) sin perder su motivación principal, a saber, que una inferencia es un proceso que ocurre entre términos ( $c f r$. Englebretsen 1996, p. 172ss).

\section{4. ¿Es la lógica de términos una lógica libre?}

Una vez expuestos estos elementos, a continuación ofrecemos un argumento a favor de que TFL se comporta como una lógica libre aunque, como veremos, no sea una lógica libre. En corto, prestamos atención a la sintaxis y a las reglas 


\begin{tabular}{|l|l|}
\hline Proposición & TFL \\
\hline \hline $\begin{array}{l}\text { 1. Algunos caballos son más rápidos } \\
\text { que algunos perros. }\end{array}$ & $+C_{1}+\left(+R_{1} 2+P_{2}\right)$ \\
\hline $\begin{array}{l}\text { 2. Los perros son más rápidos } \\
\text { que algunos hombres. }\end{array}$ & $-P_{2}+\left(+R_{2} 3+H_{3}\right)$ \\
\hline $\begin{array}{l}\text { 3. Lo que es más rápido } \\
\text { que lo que es más rápido } \\
\text { que los hombres, } \\
\text { es más rápido que los hombres. }\end{array}$ & $-\left(+R_{1} 2+\left(+R_{2} 3+H_{3}\right)\right)+\left(+R_{1} 3+H_{3}\right)$ \\
\hline $\begin{array}{l}\text { Algunos caballos son más rápidos } \\
\text { que algunos hombres. }\end{array}$ & $+C_{1}+\left(+R_{1} 3+H_{3}\right)$ \\
\hline
\end{tabular}

Tabla 5: Ejemplo con proposiciones relacionales

\begin{tabular}{|l|l|}
\hline Proposición & TFL \\
\hline \hline 1. Todo hombre es mortal. & $-\mathrm{H}+\mathrm{M}$ \\
\hline 2. Sócrates es hombre. & $+\mathrm{s}+\mathrm{H}$ \\
\hline$\vdash$ Sócrates es mortal & $+\mathrm{s}+\mathrm{M}$ \\
\hline
\end{tabular}

Tabla 6: Ejemplo con proposiciones singulares

de inferencia de TFL, las cuales descartan la aplicación irrestricta de ciertas reglas de inferencia de FOL.

Así pues, consideremos que si TFL se comporta como una lógica libre entonces deben ocurrir dos condiciones: (a) que las versiones en TFL de las reglas $E \forall$ y $I \exists$ de FOL produzcan inferencias inválidas en TFL y (b) que las versiones en TFL de las reglas $E \forall$ y $I \exists$ de FFOL produzcan inferencias válidas en TFL.

Entonces, por reductio, supongamos que TFL se comporta como una lógica libre pero que o bien (a) las versiones en TFL de las reglas $E \forall$ y $I \exists$ de FOL producen inferencias válidas en TFL o bien (b) que las versiones en TFL de las reglas $E \forall$ y $I \exists$ de FFOL producen inferencias inválidas en TFL.

Para (a) consideremos que TFL, como FFOL, descarta la aplicación irrestricta de $E \forall$ y $I \exists$. Esto se puede inferir de las expresiones equivalentes de estas reglas, las cuales son válidas en FOL pero no son válidas en TFL: 


\begin{tabular}{|l|l|}
\hline Proposición & TFL \\
\hline \hline 1. Todo hombre es mortal. & $-\mathrm{H}+\mathrm{M}$ \\
\hline 2. Sócrates es hombre. & $+\mathrm{s}+\mathrm{H}$ \\
\hline$\vdash$ Sócrates es mortal & $+\mathrm{s}+\mathrm{M}$ \\
\hline
\end{tabular}

Tabla 7: Ejemplo con proposiciones singulares

\begin{tabular}{|l|l|}
\hline Proposición & TFL \\
\hline \hline 1. Si eres Sócrates, entonces eres amigo de Platón. & $-[\mathrm{s}]+[\mathrm{p}]$ \\
\hline 2. Eres Sócrates. & $+[\mathrm{s}]$ \\
\hline$\vdash$ Eres amigo de Platón. & $+[\mathrm{p}]$ \\
\hline
\end{tabular}

Tabla 8: Ejemplo con proposiciones compuestas

En efecto, si aplicamos DON a las versiones terministas de $E \forall$ y $I \exists$ podemos verificar que la suma de las premisas no es igual a la conclusión. Luego, contra (a), las versiones en TFL de las reglas $E \forall$ y $I \exists$ de FOL no producen inferencias válidas en TFL.

Por el contrario, para (b), las reglas correspondientes a $E \forall$ y $I \exists$ en TFL se asemejan y se comportan como en FFOL:

En efecto, para la versión terminista de $E \forall$ aplicamos DON y obtenemos la expresión $(-X+A)+(+X \pm t)=-X+A+X \pm t= \pm t+A$; y para la versión terminista de $I \exists$ aplicamos DON y obtenemos la expresión $\pm t+A)+(+X \pm t)=$ $\pm t+A+X \pm t=+X+A$. Luego, contra (b), las versiones en TFL de las reglas $E \forall$ y $I \exists$ de FFOL producen inferencias válidas en TFL; y con esto obtenemos una contradicción de nuestra suposición inicial.

\begin{tabular}{|l|c|c|c|c|}
\hline Regla & FOL & ¿Válida? & TFL & ¿Válida? \\
\hline$E \forall$ & $\begin{array}{c}\forall x A x \\
\vdash A[t / x]\end{array}$ & Sí & $\begin{array}{c}-X+A \\
\vdash \pm t+A\end{array}$ & No \\
\hline$I \exists$ & $\begin{array}{c}\text { At } \\
\vdash \exists x A[t / x]\end{array}$ & Sí & $\begin{array}{c} \pm t+A \\
1 \pm X+A\end{array}$ & No \\
\hline
\end{tabular}

Figura 1: Comparación de FOL y TFL 


\begin{tabular}{|c|c|c|c|c|}
\hline Regla & FFOL & ¿Válida & TFL & ¿Válida \\
\hline$E \forall$ & $\begin{array}{l}\forall x A x \\
E ! t \\
\vdash A[t / x]\end{array}$ & Sí & $\begin{array}{l}-X+A \\
\pm t+X \\
\vdash \pm t+A\end{array}$ & Sí \\
\hline$I \exists$ & $\begin{array}{l}A t \\
E ! t \\
\vdash \exists x A[t / x]\end{array}$ & Sí & $\begin{array}{l} \pm t+A \\
\pm t+X \\
\vdash+X+A\end{array}$ & Sí \\
\hline
\end{tabular}

Tabla 9: Comparación de FFOL y TFL

\begin{tabular}{|c|c|c|c|}
\hline Ejemplo & FOL & FFOL & TFL \\
\hline Todo es bueno & $\forall x B x$ & $\forall x B x$ & $-X+B$ \\
Luego, Adso de Melk es bueno. & $\vdash B a$ & $\forall B a$ & $\forall \pm a+B$ \\
\hline Todo es bueno. & $\forall x B x$ & $\forall x B x$ & $-X+B$ \\
Adso de Melk existe. & $E ! a$ & $E ! a$ & $+X \pm a$ \\
Luego, Adso de Melk es bueno. & $\vdash B a$ & $\vdash B a$ & $\vdash \pm a+B$ \\
\hline Adso de Melk es un imbécil. & $I a$ & $I a$ & $\pm a+I$ \\
Luego, existe un imbécil. & $\vdash \exists x I x$ & $\forall \exists x I x$ & $\forall \pm a+I$ \\
\hline Adso de Melk es un imbécil. & $I a$ & $I a$ & $\pm a+I$ \\
Adso de Melk existe. & $E ! a$ & $E ! a$ & $+X \pm a$ \\
Luego, existe un imbécil. & $\vdash \exists x I x$ & $\vdash \exists x I x$ & $\vdash+X+I$ \\
\hline
\end{tabular}

Figura 2: Comparación de FOL, FFOL y TFL

Para ilustrar el argumento anterior consideremos, a modo de ilustración, los siguientes ejemplos, donde $a:=A d s o$ de Melk, Bx:=x es bueno e I $x:=x$ es un imbécil para FOL y FFOL; y $a:=A d s o$ de Melk, B:=es bueno e $I:=e s$ un imbécil para TFL:

Con todo, que TFL se comporte como FFOL con respecto a ciertas reglas de inferencia no significa que TFL sea una lógica libre. La razón por la cual TFL no puede ser una lógica libre tiene que ver con cuestiones de origen: una lógica libre es, por antonomasia, un sistema de primer orden y TFL, como decíamos, no es un sistema de primer orden, sino un sistema terminista.

Por supuesto, podríamos ampliar nuestra definición de lógica libre para admitir a TFL como una lógica libre, pero hacer tal cosa sería impreciso en 
este momento, considerando la literatura consolidada sobre lógicas libres ( $c f r$. Bencivenga, 2002) y que TFL tiene sus propios supuestos ontológicos.

\section{Conclusiones}

Así pues, concluir que TFL es una lógica libre es pedir demasiado, pues no es un sistema de primer orden; pero admitir que no se comporta como una lógica libre es corto de miras, pues su conducta inferencial es similar al de una lógica libre. Por tanto, parece justo concluir que TFL se comporta como una lógica libre aunque no sea una lógica libre. En cualquier caso, en el contexto asimétrico entre estudios sobre lógicas libres y lógicas de términos, este breve resultado ofrece cierta evidencia de que TFL es más bien una lógica tradicional que no es clásica.

\section{Apéndice A. Silogística}

La silogística es una lógica de términos que estudia la relación de inferencia entre proposiciones categóricas. Una proposición categórica es una proposición declarativa, que afirma o niega algo acerca de algo ( $P r$. An. A.1, 24a16-17), compuesta por dos términos, una cantidad y una cualidad. El sujeto y el predicado de la proposición se llaman términos ( $P r$. An. A.1, 24b16-17): el término-esquema $S$ denota el término sujeto de la proposición y el término-esquema $P$ denota el término predicado. La cantidad puede ser universal (Todo) o particular (Algún) y la cualidad puede ser afirmativa (es) o negativa (no es). Estas proposiciones categóricas se denotan mediante una etiqueta (a, para la universal afirmativa $(\mathrm{SaP})$; e, para la universal negativa $(\mathrm{SeP})$; i, para la particular afirmativa $(\mathrm{SiP})$; y o, para la particular negativa $(\mathrm{SoP}))$ que nos permite determinar una secuencia de tres proposiciones que se conoce como modo. Un silogismo categórico, entonces, es un modo ordenado de tal manera que dos proposiciones fungen como premisas y la última como conclusión (Pr. An. A.1, 24b18-20). Al interior de las premisas existe un término que ocurre en ambas premisas pero no en la conclusión: este término especial, usualmente denotado con el término-esquema $\mathrm{M}$, funciona como un enlace entre los términos restantes y es conocido como término medio. De acuerdo a la posición del término medio se pueden definir cuatro arreglos o figuras que codifican los modos o patrones silogísticos correctos (Tabla 8 ). 


\begin{tabular}{|l|l|l|l|}
\hline Primera figura & Segunda figura & Tercera figura & Cuarta figura \\
\hline aaa Barbara $)$ & eae $($ Cesare $)$ & iai $($ Disamis $)$ & aee $($ Calemes $)$ \\
\hline aea $($ Celarent $)$ & aee $($ Camestres $)$ & aii $($ Datisi $)$ & iai $($ Dimaris $)$ \\
\hline aii $($ Darii $)$ & eio $($ Festino $)$ & oao $($ Bocardo $)$ & eio Fresison $)$ \\
\hline eio $($ Ferio $)$ & aoo (Baroco $)$ & eio $($ Ferison $)$ & \\
\hline
\end{tabular}

Tabla 10: Modos silogísticos correctos

\section{Referencias}

Bencivenga, E. (2002), "Free Logics”, en D.M. Gabbay y F. Guenthner 2002, pp. 147-196.

Englebretsen, G. (1996), Something to Reckon with: The Logic of Terms, University of Ottawa Press, Canadá.

Fenstad, J. E. (1971), Proceedings of the Second Scandinavian Logic Symposium, North-Holland, Ámsterdam.

Gabbay, D.M. y Guenthner, F. (2002), Handbook of Philosophical Logic, 2a edición, vol. 5, Kluwer, Dordrecht.

Hailperin, Th. (1953), "Quantification Theory and Empty Individual Domains", Journal of Symbolic Logic, vol. 18, pp. 197-200.

Jaśkowski, S. (1934), "On the Rules of Supposition in Formal Logic," Studia Logica, vol. 1 , pp. 5-32

Kuhn, S.T. (1983), "An Axiomatization of Predicate Functor Logic", Notre Dame Journal of Formal Logic, vol. 24, no. 2, pp. 233-241.

Lambert, K. (1958), "Notes on E!", Philosophical Studies, vol. 9, pp. 60-63.

Lambert, K. (1960). "The Definition of E! in Free Logic", en Abstracts: The International Congress for Logic, Methodology and Philosophy of Science, Stanford University Press, Stanford.

Lambert, K. (1963), "Existential Import Revisited", Notre Dame Journal of Formal Logic, vol. 4, pp. 288-292.

Lejewski, C. (1954) "Logic and Existence", British Journal for the Philosophy of Science, vol. 5, no. 18, pp. 104-19.

Leonard, H. S. (1956), "The Logic of Existence", Philosophical Studies, vol. 7, pp. 49-64.

Mostowski, A. (1951), "On the Rules of Proof in the Pure Functional Calculus of the First Order", Journal of Symbolic Logic, vol. 16, pp. 107-111.

Noah, A.A. (1980), "Predicate-functors and the Limits of Decidability in Logic", Notre Dame Journal of Formal Logic, vol. 21, no. 4, pp 701-707.

Oderberg, D. (2005), "Predicate Logic and Bare Particulars", en D. Oderberg 2005, pp. 183-210. 
Oderberg, D. (2005), The Old New Logic: Essays on the Philosophy of Fred Sommers, MIT Press, Cambridge.

Palau, G. (2002), Introducción filosófica a las lógicas no-clásicas, Gedisa.

Quine, W.V.O. (1971), "Predicate Functor Logic”, en J. E. Fenstad 1971, pp. 309-315.

Sommers, F. (1975), "Distribution Matters", Mind, vol. 84, no. 1, pp. 27-46.

Sommers, F. (1984), The Logic of Natural Language, Oxford University Press, Nueva York.

Sommers, F. (1989), "Predication in the Logic Terms", Notre Dame Journal of Formal Logic, vol. 31, no. 1, pp. 106-126.

Sommers, F. (2005), "Intelectual Autobiography", en D. Oderberg 2005, pp. 1-23.

Sommers, F. y Englebretsen, G. (2017), An Invitation to Formal Reasoning: the Logic of Terms, Routledge, Londres.

Woods, J. y Irvine, A. (2004), “Aristotle's Early Logic”, en J. Woods y D.M. Gabbay 2004, pp. 27-99.

Woods, J. y Gabbay, D.M. (2004), Handbook of the History of Logic, vol. 1, Elsevier. 